\title{
Preparation of Metallic Films by Electrodeposition at the Interface between Two Liquid Phases
}

\author{
Hiroyuki Kaneko, Hitoshi Taimatsu and Hisashi Sakahara \\ Department of Materials Engineering and Applied Chemistry, Mining College, Akita University, \\ Akita 010, Japan
}

\begin{abstract}
A novel technique is described for continuously obtaining metallic films: a metallic film is produced by electrolysis at the interface between an organic liquid and an aqueous solution of a metal salt, and the film growing uni-directionally along the interface is rolled onto a rotating drum. As an example, zinc films are grown at the n-butyl acetate/zinc sulfate solution interface. The zinc films have a thickness of about $1 \mu \mathrm{m}$, and their growth directions are parallel to the basal plane of zinc. Some factors governing the metallic film formation are discussed.
\end{abstract}

(Received April 2, 1993)

Keywords: electrodeposition, interfacial phenomena, metallic films, filmy growth, butylacetate, zinc, zinc sulfate

\section{Introduction}

Thin metallic films are important in numerous industrial applications, especially in electronic and magnetic devices. One of their preparation methods is the electrodeposition of a metal on a substrate from an aqueous solution. In this method, very thin films can not easily be obtained because of the difficulty of detaching from the substrate. In the paper of Mylius and Fromm ${ }^{(1)}$ one century ago on the two-dimensional metal deposition at the interface between two liquid phases, there is a hint to overcome this disadvantage.

Two-dimensional metal deposits, the so-called "metalleaves," can be obtained by the cathodic reduction of metal ions at an interface between an organic liquid and an aqueous solution of the metal salt under appropriate experimental conditions ${ }^{(1)(2)}$. A similar phenomenon was

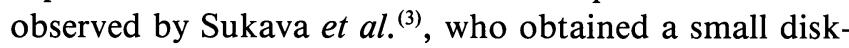
like copper deposit grown at the interface between air and an aqueous solution containing a small amount of an organic surfactant. Sawada et al. ${ }^{(4)}$ and Greier et al. $.^{(5)} \mathrm{ob}-$ tained spoke-like metal-leaves in cells, called Hell-Shaw cells, which consisted of two closely-spaced glass plates sandwiching a thin layer of an aqueous solution. One of the present authors ${ }^{(6)}$ investigated the morphology of zinc leaves growing at a point cathode at the interface butyl acetate/zinc sulfate solution. Two types of deposits were obtained: one was spoke-like and the other was filmy. It was shown that the filmy growth was favored at lower current densities and the spoke-like growth at higher current densities. Several further experimental conditions for a filmy deposit to become a concentric circle at the tip of a cathodic wire were in- vestigated ${ }^{(7)}$.

Recently the present authors succeeded in preparing uni-directionally growing metal films of copper, cobalt, zinc and silver. In this paper, the preparation method of zinc films at the interface between two liquid phases is presented and their morphologies are metallographically examined.

\section{Experimental}

The apparatus used is shown in Fig. 1. The electrolytic cell was a glass box, $26 \mathrm{~mm}$ wide, $50 \mathrm{~mm}$ deep, and 30 $\mathrm{mm}$ high, with a water-jacket to circulate thermostated water. The cell was filled with an aqueous zinc sulfate solution of $3 \mathrm{kmol} \cdot \mathrm{m}^{-3}$ covered with $\mathrm{n}$-butyl acetate. The cathode was an aluminum foil, $10 \mathrm{~mm}$ wide and 15 $\mu \mathrm{m}$ thick, floating at the interface between the aqueous solution and n-butyl acetate; its one end out of the cell was connected to the rotating drum. The anode was a zinc plate, $26 \mathrm{~mm}$ deep, and $1 \mathrm{~mm}$ thick, and was

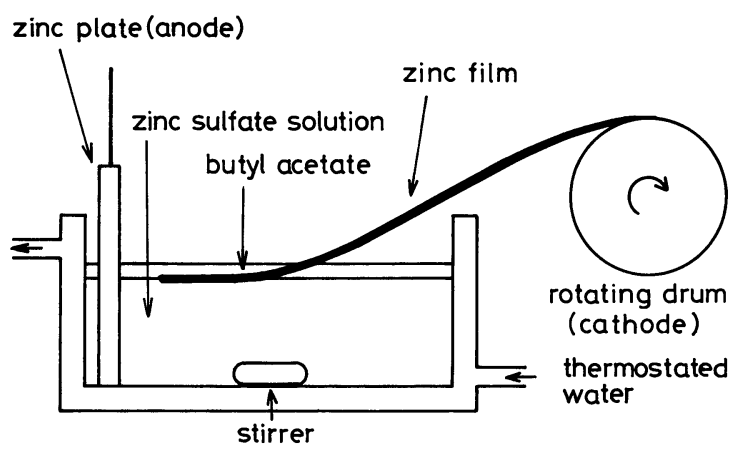

Fig. 1 Apparatus for preparing metallic films. 
positioned vertically to the cathode. Cathodic reduction of zinc ions was conducted with a potentio-galvanostat (Hokuto Denko, HA501G), and a zinc film growing along the interface was continuously rolled onto the drum by a computer-controlled stepping motor so that the distance between the anode and the growing front of the zinc film was kept constant at $5 \mathrm{~mm}$.

\section{Results and Discussion}

The growth morphology depended on several factors. Of these, the most important was the choice of an adequate organic liquid. Without organic liquid, i.e., at the air/aqueous solution interface, the filmy growth of zinc was not observed. The solubility of organic liquids in aqueous solutions seems to play an important role. Highly hydrophiric liquids were inadequate to form the stable interfaces with aqueous solutions. Highly hydrophobic liquids such as silicon oil also failed to give metal films. Acetic esters such as iso-butyl acetate, namyl acetate and n-hexyl acetate were good for preparing zinc films, as well as n-butyl acetate used in the present study $^{(8)}$.

Temperature and current were also critical. At the nbutyl acetate/zinc sulfate solution interface, it was not easy to obtain a filmy deposit at temperatures below $20^{\circ} \mathrm{C}$. With increasing temperature, however, one side of the filmy deposit facing to the aqueous solution was covered with muddy deposits. Although the current needed depended on the cell configulation, at high applied currents above $200 \mathrm{~mA}$, a spoke-like deposit, not a filmy one, grew. At low applied currents below $50 \mathrm{~mA}$, any films did not grow. Therefore, the filmy growth was favored at higher temperatures than room temperature and in a limited range of applied current.

In the controlled-current electrolysis, the applied voltage became increased with time due to the concentration polarization. The concentration polarization could be reduced by circulating the solution during the electrolysis. Under the controlled-voltage electrolysis, the current decreased with time and the film growth ceased in due time. Therefore, the controlled-current electrolysis is superior to the controlled-voltage electrolysis to continue the film growth. The growth morphology of a zinc film produced by the controlled-current electrolysis at $80 \mathrm{~mA}$ at $30^{\circ} \mathrm{C}$ is shown in Fig. 2. At a current of $80 \mathrm{~mA}$, a zinc film $25 \mathrm{~mm}$ wide grew uni-directionally at a constant growth rate of $4 \mathrm{~mm} / \mathrm{min}$ from the tip of the aluminum foil. From the weight of the deposit and the quantity of electricity passed, it was shown that the current efficiency was practically $100 \%$. The current was spent on the filmy growth along the interface and the thickning of the film. The as-grown film had different surfaces in appearance: one facing toward the organic liquid side was lustrous and covered thinly with fine down of zinc, and the other facing toward the aqueous solution side was dark grey as shown in Fig. 3 and covered with aggregates of fine spokes of zinc. These surface morphilogies are similar to those obtained at the $n$-hexyl acetate $/ \mathrm{ZnSO}_{4}$ aqueous
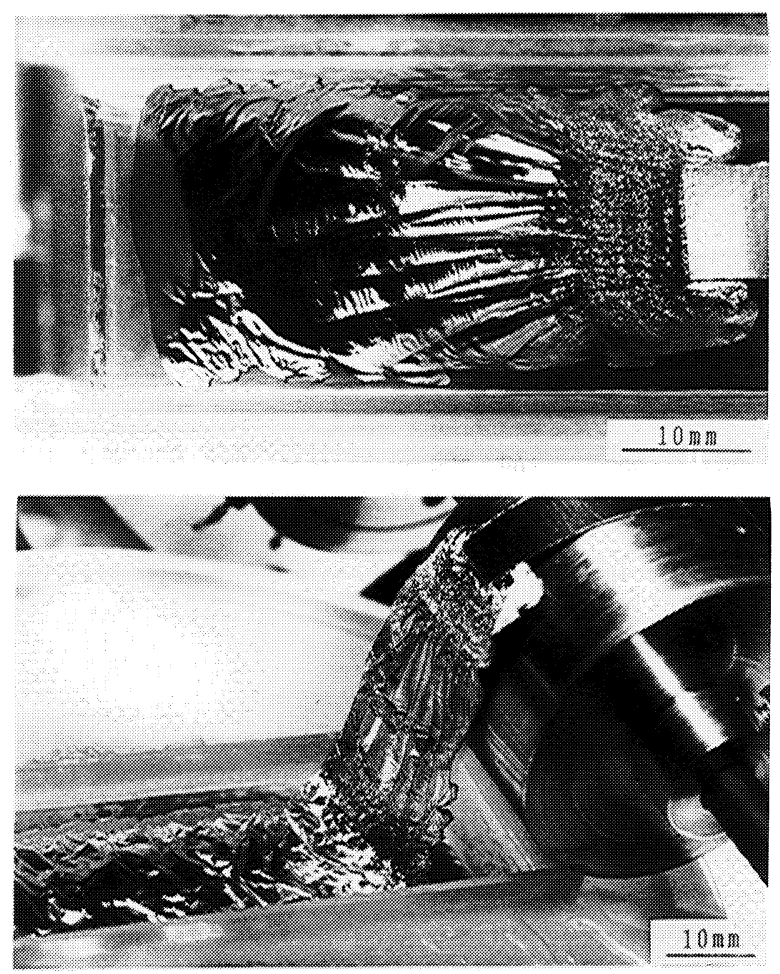

Fig. 2 Growth of a zinc film.

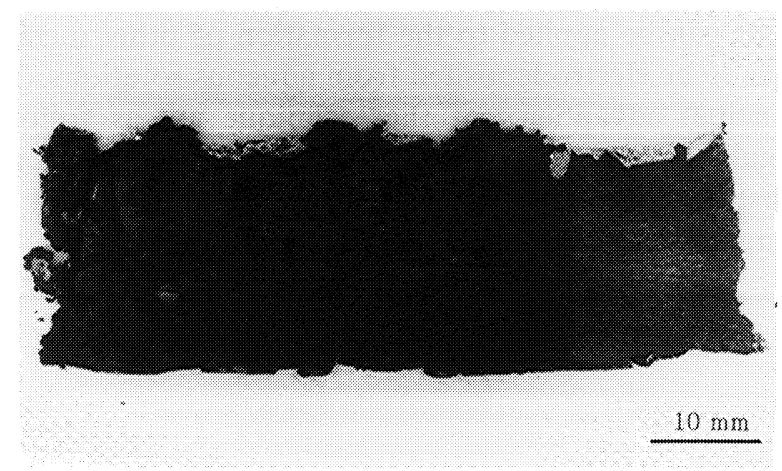

Fig. 3 Morphology of a film surface facing toward the aqueous solution side.

solution interface ${ }^{(7)}$. The thickness of the film itself was about $1 \mu \mathrm{m}$ everywhere. X-ray diffraction patterns using $\mathrm{Cu} \cdot K_{\alpha}$-ray are shown in Fig. 4 . As-grown film has several diffraction lines by deposits on the film surface (Fig. 4(a)), but the film itself, from which deposits were removed by washing with a dilute $\mathrm{HCl}$ solution, has only simple diffraction lines from the basal plane (Fig. 4(b)). TEM observation showed that the deposits on the aqueous solution side consisted of hexagonal platelets. This structure is analogous to that of zinc deposits obtained by Noguchi et $a l .{ }^{(9)}$ in zinc sulfate solutions.

The present authors now investigate the reason why metallic films grow at the interfaces between organic liquids and aqueous solutions. The growth of metallic deposit at the interface, irrespective of whether it is spoke-like or filmy, mostly occurred when the interfacial 


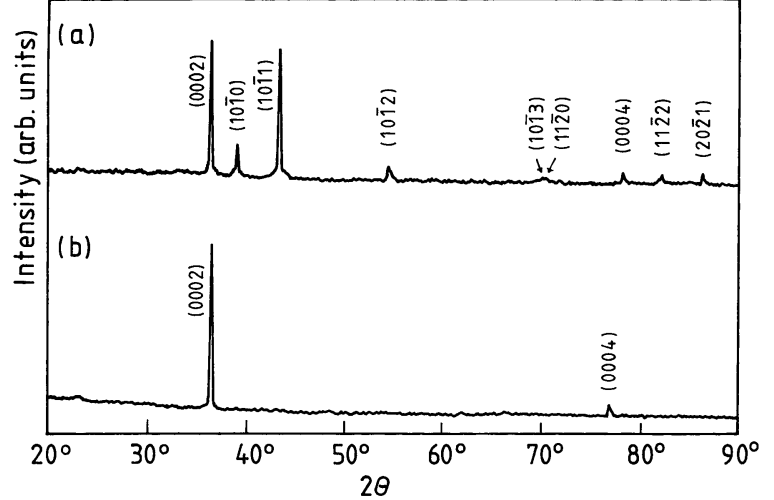

Fig. 4 X-ray diffraction patterns of a film $\left(\mathrm{Cu} \cdot K_{\alpha}\right)$ : (a) as-grown and (b) washed with a dilute $\mathrm{HCl}$ solution.

tension between an organic liquid and an aqueous solution is high. The growth rate of a deposit, i.e., current density, probably determines its morphology. Rapid and slow growth results in spoke-like and filmy morphology, corresponding to the growth of dendrites and layers in the electrocrystallization of metals in aqueous solutions ${ }^{(10)}$. Further details will be presented elsewhere.

The technique developed in the present study was successfully applied to the formation of copper cobalt, and silver films about $1 \mu \mathrm{m}$ thick, as well as zinc. This technique can be applied to the formation of other metallic films to be electrodeposited from aqueous solutions. The principal advantages of the technique are as follows: metallic films are easily obtainable without using any substrate for deposition, and the crystal direction of the film can be controlled.

\section{Conclusion}

An electrochemical technique for producing metallic thin films has been developed and applied to the preparation of zinc films. The zinc film obtained by the electrolysis at the interface between n-butyl acetate and a zinc sulfate solution is continuously rolled onto the rotating drum. Zinc films about $1 \mu \mathrm{m}$ in thickness with the growth direction along the basal plane of zinc was obtained. Filmy growth of zinc is favored at high temperatures and at low applied currents or voltages. By this technique, thin metallic films grown uni-directionally can be obtained without using substrates for deposition.

\section{Acknowledgment}

We thank Dr. R. Tamamushi at the Institute of Physical and Chemical Research for his helpful discussions during this work. The present work was partially supported by a Grant-in-Aid for Scientific Research (No. 03805059) from the Ministry of Education, Science and Culture of Japan.

\section{REFERENCES}

(1) F. Mylius and O. Fromm: Ann. Phys. Chem., 51 (1894), 593.

(2) H. Freundrich and W. Novikov: Z. Elektrochem., 16 (1910), 394.

(3) A. J. Sukava, W. J. Newby, R. A. Diolorio, R. N. Sharms, K. P. Chu: Nature, 228 (1968), 574.

(4) Y. Sawada, A. Dougherty, J. P. Gollub: Phys. Rev. Lett., 56 (1986), 1260.

(5) D. Grier, E. Ben-Jacob, R. Clark, L. M. Sander: Phys. Rev. Lett., 56 (1986), 1264.

(6) R. Tamamushi and H. Kaneko: Electrochim. Acta, 25 (1980), 391.

(7) H. Kaneko, H. Sakahara and H. Taimatsu: J. Mining and Materials Processing Inst. Jpn., 107 (1991), 542.

(8) H. Sakahara, H. Kaneko and H. Taimatsu: Hyomen Gijutsu, 43 (1992), 1059.

(9) F. Noguchi, K. Kaneko, M. Kikuchi: Denki Kagaku, 43 (1975), 84.

(10) G. Wranglen, Electrochim. Acta: 2 (1960), 130. 